\title{
New Speech Compression Technique based on Filter Bank Design and Psychoacoustic Model
}

\author{
Mourad Talbi \\ Laboratory of Semi-Conductors, Nanostructures and Advanced Technology, ${ }^{1}$ Center of Researches and Technolo- \\ gies of Energy of Borj Cedria, B.P N95 2050 - Hammam Lif - Tunis, Tunisia.
}

\author{
Med Salim Bouhlel \\ Sciences Electroniques, Technologie de l'Information et Télécommunications (SETIT), Sfax BP 11693029, Tunisia.
}

(Received 13 November 2017; accepted 21 March 2019)

\begin{abstract}
In this paper, a new speech compression technique is proposed. This technique applies a Psychoacoustic Model and a general approach for Filter Bank Design using optimization. It is evaluated and compared with a compression technique using a MDCT (Modified Discrete Cosine Transform) Filter Bank of 32 Filters and a Psychoacoustic Model. This evaluation and comparison is performed by calculating bits before and after compression, PSNR (Peak Signal to Noise Ratio), NRMSE (Normalized Root Mean Square Error), SNR (Signal to Noise Ratio) and PESQ (Perceptual evaluation of speech quality) computations. The two techniques are tested and applied to a number of speech signals that are sampled at $8 \mathrm{kHz}$. The results obtained from this evaluation show that the proposed technique outperforms the second compression technique (based on a Psychoacoustic Model and MDCT filter Bank) in terms of Bits after compression and compression ratio. In fact, the proposed technique yields higher values for the compression ratio than the second compression technique. Moreover, the proposed compression technique presents reconstructed speech signals with acceptable perceptual qualities. This is justified by the values of SNR, PSNR and NRMSE and PESQ.
\end{abstract}

\section{INTRODUCTION}

The principal aim of speech compression is to represent, with the minimum number of bits, the digital speech waveform while at the same time preserving its perceptual quality. ${ }^{1,2}$ The compression of speech signals is needed for both reducing memory storage requirements and for reducing transmission bandwidth requirements while also preserving speech quality. ${ }^{3}$ For example, digital cellular phones employ some compression approaches in order to compress in real-time the speech signal over general switched telephone networks. Compression is also applied in order to reduce the storage requirements for storing the voice messages or for mail forwarding of voice messages. All these applications depend on the effectiveness of the speech compression approach. Thus, in the past, different approaches were developed in order to meet the rising demand for better techniques of speech compression. ${ }^{4}$ In general, speech compression techniques can be grouped into two classes. These classes are Lossy compression and Lossless compression. The latter is often performed by waveform coding techniques. For Lossless compression, the actual shape of the signal produced by the microphone and its associated circuits is preserved. ${ }^{5}$ The most well-known waveform coding method is Pulse Code Modulation (PCM). Others Lossless approaches make speech signals compression by localizing redundancy and optimizing, or suppressing, it by the quantization process. Among these approaches, we can mention differential quantization and adaptive PCM. All such techniques require simple signal processing and lead to a minimum distor- tion and small values for the compression ratio. ${ }^{6,7}$ A detailed study on these techniques was presented in references ${ }^{7}$ and. ${ }^{8}$ Concerning Lossy compression, the compressed data is a close approximation of the original data. However, it gives much higher values for the compression ratio than lossless compression. The literature review reveals that substantial progress has been made on Lossy compression approaches such as Linear Predictive Coding (LPC), ${ }^{4}$ sub-band coding ${ }^{8}$ and turning point. ${ }^{5}$ When making sub-band decomposition of the original signal, its spectral information is divided into a set of signals that can then be encoded by employing a variety of approaches. Based on sub-band decomposition, different methods have been devised for speech compression. ${ }^{9}$ In this context, Adaptive Speech Compression Based on Discrete Wave Atoms Transform, was proposed by Souha et al. ${ }^{10}$ The first step of this technique consists of decomposing the speech signal into wave atoms. ${ }^{10}$ In the second step, the obtained wave atom coefficients are truncated using an adaptive thresholding. The latter depends on the SNR estimation. The threshold coefficients are then quantized using the Max Lloyd scalar quantizer. Furthermore, they are encoded using zero run-length encoding followed by Huffman coding. In this paper, we propose a new speech compression technique that is based on both a Psychoacoustic Model and a general approach for Filter Bank Design using optimization. It consists of replacing the MDCT (Modified Discrete Cosine Transform) filter bank of 32 filters, which was used in the compression system proposed by Alex et al., ${ }^{11-13}$ with both a uniform and non-uniform filter bank designed using optimization. ${ }^{14,15}$ 
For the rest of this paper, section 2 is devoted to background on the Psychoacoustic Model. In section 3, we will deal with a general approach for Filter Bank Design using Optimization. In section 4, we will detail the proposed compression technique. In section 5, we will deal with the performance evaluation. Section 6 is devoted to File Format and Compression. Section 7 presents results and discussion. Finally, in section 8 , we will conclude.

\section{BACKGROUND ON PSYCHOACOUSTIC MODEL}

The Psychoacoustic Model was based on research that focuses on human perception. Those works proved that the average human hearing for all frequencies is not the same. Effects due to the limitations of the human sensory system and diverse sounds in the environment lead to the facts that can be used for eliminating unnecessary data in an audio signal. ${ }^{11-13}$ The two essential properties of the human auditory system that make up the Psychoacoustic Model are the hearing absolute threshold and auditory masking. Each of them provides a way of determining which signal zones are indiscernible and inaudible to the average human and can consequently be removed from the original signal. ${ }^{11-13}$

\section{A GENERAL APPROACH FOR FILTER BANK DESIGN USING OPTIMIZATION}

In speech processing, different applications use Filter-Banks (Fig. 1). ${ }^{14,15}$ One of the main necessities in Filter bank design is the Perfect Reconstruction (PR). This means that the filter bank can't introduce some distortions on the signal. The filter bank can generally be classified into two main classes. The first one is of uniform filter banks where all sampling rates, $\left\{n_{1}, n_{2}, \ldots, n_{K}\right\}$ are similar. The second class is of nonuniform filter banks, where at least one sampling rate does not equal to the others. ${ }^{14,15} \mathrm{In}^{14,15}$, and the references therein, there was a detailed general approach for Filter Bank Design employing Optimization.

\subsection{The Perfect Reconstruction Conditions}

Consider a filter bank owing $\left\{n_{1}, n_{2}, \ldots, n_{K}\right\}$ as the integer sampling rates (Fig. 1). The filter bank output in the Z-domain, was formulated as follows: ${ }^{14,15}$

$$
\begin{aligned}
\hat{X}(z)= & \sum_{k=1}^{K} \frac{1}{n_{k}} F_{k}(z) \sum_{l=0}^{n_{k}-1} X\left(z e^{-j \frac{2 \pi l}{n_{k}}}\right) H_{k}\left(z e^{-j \frac{2 \pi l}{n_{k}}}\right) \\
= & \sum_{k=1}^{K} \frac{1}{n_{k}} F_{k}(z) X(z) H_{k}(z) \\
& +\sum_{k=1}^{K} \frac{1}{n_{k}} F_{k}(z) \sum_{l=1}^{n_{k}-1} X\left(z e^{-j \frac{2 \pi l}{n_{k}}}\right) H_{k}\left(z e^{-j \frac{2 \pi l}{n_{k}}}\right) \\
= & X(z) \cdot T_{0}(z)+\sum_{l=1}^{n_{k}-1} X\left(z e^{-j \frac{2 \pi l}{n_{k}}}\right) \cdot T_{1}(z)
\end{aligned}
$$

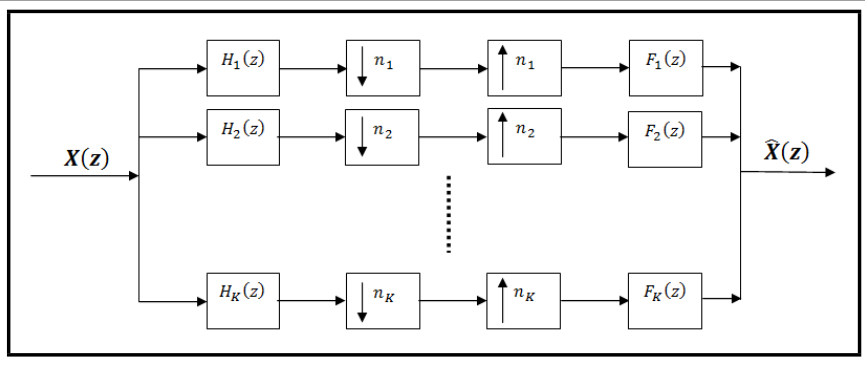

Figure 1. A General form of Filter bank. ${ }^{14}$

where we had:

$$
\begin{aligned}
& T_{0}(z)=\sum_{k=1}^{K} \frac{1}{n_{k}} F_{k}(z) H_{k}(z) ; \\
& T_{1}(z)=\sum_{k=1}^{K} \frac{1}{n_{k}} F_{k}(z) H_{k}\left(z e^{-j \frac{2 \pi l}{n_{k}}}\right) \quad \text { for } \quad l=1, \ldots, K ;
\end{aligned}
$$

where both $T_{0}(z)$ and $T_{1}(z), l \neq 0$, designated the overall distortion transfer function and the aliasing transfer function corresponding to $X\left(z e^{-j \frac{2 \pi l}{n_{k}}}\right)$. If $T_{1}(z)$ was zero and $T_{0}(z)$ was a pure delay, then we obtained PR. ${ }^{14,15}$

\subsection{Filter Bank Design Algorithm}

As previously mentioned, the filter bank is used for splitting a signal into a number of frequency sub-bands by using analysis filters. Following this, each of these sub-bands are processed separately. Consequently, the first requirement is to have the analysis filters $H_{k}(z)$ satisfy some prescribed frequency specifications. The second requirement is that the analysis and synthesis filters satisfy the PR condition. ${ }^{14,15} \mathrm{In}^{14,15}$, the goal was to conceive a filter bank that achieves $\mathrm{PR}^{14}$ and the Magnitude Response of the Analysis Filters (MRAF) satisfied some prescribed requirements. $\operatorname{In}^{14,15}$, just the MRAF was considered. The phase response of each individual analysis filter was ignored for increasing the degrees of freedom for a given length $N$. Although each designed filter may not have had a linear phase, the Perfect Reconstruction conditions ensured the linear phase of the whole filter bank. The design process needed to find the analysis and synthesis filter coefficients $\left\{h_{k}, f_{k}\right\}$ so that the Perfect Reconstruction conditions detailed in ${ }^{14,15}$ and the prescribed specifications for MRAF were satisfied. The design method was based on minimizing the following performance index, which combines $e_{P R}$ (the Perfect Reconstruction error) and $e_{F}$ (the magnitude response error), with respect to analysis filters coefficients:

$$
J=e_{P R}+e_{F}=W_{P R}\|A x-b\|^{2}+W_{F} \sum_{k=1}^{K} e_{F, k} ;
$$

where $\|\cdot\|$ was $l_{2}$-norm and $W_{F}$ and $W_{P R}$ designated the optional weights. The expressions $e_{F, k}, b, A$ and $x$ were detailed in. ${ }^{14,15}$

The optimization parameters were the analysis filters coefficients. The synthesis filters coefficients were obtained as the least square solution of the following expression:

$$
A x=b .
$$




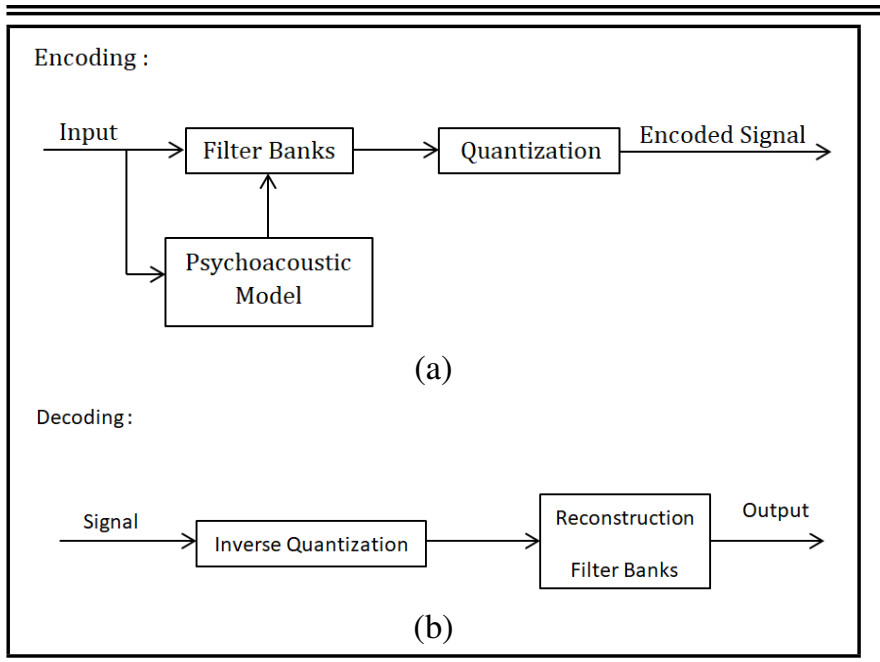

Figure 2. (a) Signal Encoding, (b) Signal Decoding.

\section{THE PROPOSED SPEECH COMPRESSION TECHNIQUE}

Alex et al. ${ }^{11-13}$ implemented a compression method by employing psychoacoustic modeling for determining which portions of the audio signal to eliminate without losing sound quality to the human ear. In this compression method, ${ }^{11-13}$ the original signal was run through a MDCT Filter Bank of 32 filters (with Perfect Reconstruction). The impulse responses $h$ and $g$ associated to this MDCT filter bank, were expressed as follow: ${ }^{11-13}$

$$
\begin{aligned}
& h(k, n)= w(n) \sqrt{\frac{2}{M}} \\
& \cos \left((2(n-1)+M+1) \frac{(2(k-1)+1) \pi}{4 M}\right) ; \\
& g(k, L-n+1)=h(k, n)
\end{aligned}
$$

with:

$$
\begin{aligned}
& 1 \leq k \leq M, 1 \leq n \leq L, L=2 M, M=32, \\
& w(n)=\sin \left(\frac{\pi}{2 M} \cdot(n-0.5)\right) .
\end{aligned}
$$

First, the signal was divided by the filter bank into separate frequency components. Following this, each of these components were quantized with a variable number of bits. This was based on the Psychoacoustic Model results. Alex et al. ${ }^{11-13}$ have analysed this compressed version of the signal and, by employing different quantization schemes, were able to achieve 30 to $75 \%$ compression of the original signal. This difference was due to the overhead needed for decoding the quantized signal in each scheme. Figure 2 shows a simplified block diagram of the compression system of Alex et al. ${ }^{11-13}$

In this paper, we replaced the MDCT filter bank of 32 filters with both a uniform and non-uniform filter bank. As previously mentioned, uniform and non-uniform filter banks are designed by using optimization. ${ }^{14,15}$ The aim was to design $\mathrm{M}$ analysis and synthesis FIR filters so that the analysis filters satisfied some frequency specifications and the filter bank (almost) met the PR conditions. This aim was achieved by minimizing the performance index, which is expressed as fol-

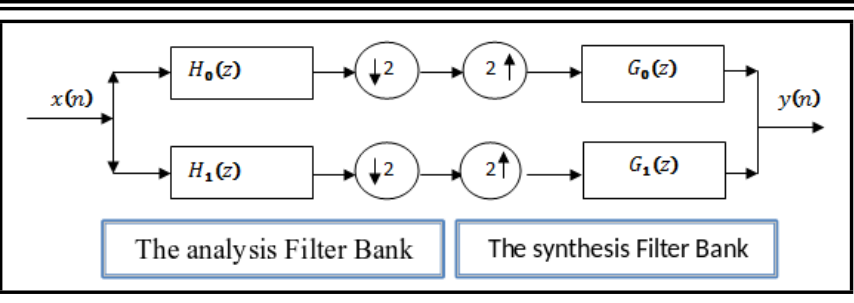

Figure 3. The two used Analysis-Synthesis optimized filter bank in this work.

Table 1. Coefficients of Analysis-Synthesis impulses responses of the designed filter bank using optimization.

\begin{tabular}{|c|c|c|c|}
\hline$h_{0}$ & $h_{1}$ & $g_{0}$ & $g_{1}$ \\
\hline-0.0052 & 0.0005 & 0.0004 & 0.0023 \\
-0.0023 & 0.0002 & 0.0002 & -0.0011 \\
0.0479 & -0.0196 & 0.0040 & 0.0882 \\
-0.0047 & -0.0060 & 0.0004 & -0.0498 \\
-0.0863 & 0.1729 & -0.2292 & -0.0377 \\
0.1611 & -0.0170 & 0.1119 & -0.4325 \\
0.5151 & -0.3864 & 0.8658 & 1.0458 \\
0.4322 & 0.5188 & 1.0158 & -0.7691 \\
0.0513 & -0.2195 & 0.3254 & -0.0450 \\
-0.1103 & -0.0171 & -0.1663 & 0.3356 \\
0.0001 & -0.0221 & -0.0152 & -0.0101 \\
0.0002 & 0.0455 & 0.0982 & -0.0348 \\
0.0000 & -0.0003 & -0.0040 & 0.0002 \\
0.0003 & 0.0014 & -0.0104 & 0.0006 \\
\hline
\end{tabular}

lows: ${ }^{14,15}$

$$
\begin{aligned}
J= & w_{1} \cdot(\text { PRerror }) \\
& +w_{2} \cdot(\text { Frequency Specification error })
\end{aligned}
$$

$w_{1}$ and $w_{2}$ represented the optional weights.

The algorithm detailed in ${ }^{15}$ can design both uniform (critically/over sampled) and non-uniform filter banks. ${ }^{14,15}$ Figure 3 illustrates the uniform Filter bank used in this work.

As shown in Figure 3, the functions $H_{0}(z), H_{1}(z), G_{0}(z)$ and $G_{1}(z)$ were respectively the Z-transforms of the impulse responses of the analysis and synthesis filters $h_{0}(n), h_{1}(n)$, $g_{0}(n)$ and $g_{1}(n)$. These impulses were determined by optimization, which was performed by minimizing the performance index given by equation (8). ${ }^{14,15}$ Therefore, in this work, we have replaced the impulse responses $h$ and $g$ associated with MDCT filters bank of 32 filters (expressed by equations (6) and (7)) by the $h_{0}, h_{1}, g_{0}$ and $g_{1}$. Table 1 shows the coefficients of these impulse responses.

The two impulse responses $h_{0}$ and $h_{1}$ were designed for analysis and the two others, $g_{0}$ and $g_{1}$ were designed for synthesis. In this paper, there were two things that justified the use of both a uniform and non-uniform Filter Bank. ${ }^{14,15}$ First, we want to reduce the calculation complexity in our speech compression system. The second one is that both the uniform and non-uniform Filter Bank is with PR.

\section{PERFORMANCE EVALUATION}

As previously mentioned, this paper evaluates the performance of our speech compression technique by comparing it with the compression method of Alex et al. ${ }^{1-13}$ This comparison is performed via the calculation of Bits before and after compression, the SNR (Signal to Noise Ratio), the PSNR (Peak Signal to Noise Ratio), the NRMSE (Normalized Root 
Table 2. Bits before and after compression for the proposed compression technique and that of Alex et al. ${ }^{11-13}$

\begin{tabular}{|c|c|c|c|c|c|c|c|}
\hline $\begin{array}{l}\text { The Compression } \\
\text { Technique }\end{array}$ & Signal & $\begin{array}{l}\text { Full Range } \\
\text { Quantization }\end{array}$ & $\begin{array}{l}\text { Narrow Range } \\
\text { Quantization }\end{array}$ & $\begin{array}{l}8 \text { bits } \\
\text { Quantization }\end{array}$ & $\begin{array}{l}16 \text { bits } \\
\text { Quantization }\end{array}$ & $\begin{array}{l}\text { Original } 16^{*} \text { number } \\
\text { of samples }\end{array}$ & $\begin{array}{l}\text { Original } \\
\text {.wav }\end{array}$ \\
\hline $\begin{array}{l}\text { Technique of } \\
\text { Alex et al. }{ }^{11-13}\end{array}$ & $\begin{array}{l}\text { Signal1: "1.wav" } \\
\text { Signal2: "2.wav" } \\
\text { Signal3: "3.wav" }\end{array}$ & $\begin{array}{l}325632 \\
298496 \\
339200\end{array}$ & $\begin{array}{l}365568 \\
335104 \\
380800\end{array}$ & $\begin{array}{l}202752 \\
185856 \\
211200\end{array}$ & $\begin{array}{l}399360 \\
366080 \\
416000\end{array}$ & $\begin{array}{l}373152 \\
385088 \\
345680\end{array}$ & $\begin{array}{l}372736 \\
385024 \\
345700\end{array}$ \\
\hline $\begin{array}{l}\text { The proposed } \\
\text { Technique }\end{array}$ & $\begin{array}{l}\text { Signal1: "1.wav" } \\
\text { Signal2: "2.wav" } \\
\text { Signal3: "3.wav" }\end{array}$ & $\begin{array}{l}265320 \\
247632 \\
247632\end{array}$ & $\begin{array}{l}279556 \\
279556 \\
249816\end{array}$ & $\begin{array}{l}184680 \\
192888 \\
172368\end{array}$ & $\begin{array}{l}369000 \\
385400 \\
344400\end{array}$ & $\begin{array}{l}373152 \\
385088 \\
345680\end{array}$ & $\begin{array}{l}372736 \\
385024 \\
345700\end{array}$ \\
\hline
\end{tabular}

Mean Square Error) and the PESQ (Perceptual Evaluation Speech Quality). ${ }^{16,17}$

\subsection{Perceptual Evaluation Speech Quality (PESQ)}

The PESQ algorithm is an objective quality measure that was approved as the ITU-T recommendation P.862. The PESQ algorithm is a tool of objective measurement that was conceived to predict the results of a subjective Mean Opinion Score (MOS) test. It was proven that PESQ is more reliable and presents a better correlation with MOS than other conventional objective speech measures. ${ }^{18}$ The PESQ score can vary in the range from -0.5 to 4.5 . A higher score means a better speech quality.

\subsection{Signal to Noise Ratio}

The SNR was expressed as follows:

$$
S N R(d B)=10 \cdot \log _{10}\left(\frac{\sum_{n=0}^{N-1} s^{2}(n)}{\sum_{n=0}^{N-1}(\hat{s}(n)-s(n))^{2}}\right) ;
$$

where the $s(n), \hat{s}(n)$ and $N$ were respectively the original signal, the processed signal and the samples number in $s(n)$.

\subsection{Normalized Root Mean Square Error (NRMSE)}

The NRMSE was expressed as follow:

$$
N R M S E=\sqrt{\frac{(s(n)-\hat{s}(n))^{2}}{\left(s(n)-\mu_{s}(n)\right)^{2}}} ;
$$

where $\mu_{s}(n)$ was the mean of the original signal $s(n)$ and $\hat{s}(n)$ was the processed signal.

\subsection{Peak Signal to Noise Ratio (PSNR)}

The PSNR was expressed as follow:

$$
P S N R=10 \cdot \log _{10}\left(\frac{N-s^{2}(n)}{\|\left(s(n)-\hat{s}(n) \|^{2}\right.}\right) ;
$$

\section{FILE FORMAT AND COMPRESSION}

For computing the compression ratios for the two compression techniques (the proposed technique and that of Alex et al. ${ }^{11-13}$ ) we first determined the number of bits that each file takes. We used the same calculation rules for file size: original files size, 16-bit quantization, 8-bit quantization, full range quantization and narrow range quantization as employed by Alex et al. ${ }^{11-13}$

\begin{tabular}{|c|c|c|c|}
\hline $\begin{array}{l}\text { The } \\
\text { Compression } \\
\text { Technique }\end{array}$ & Signal & $\begin{array}{l}\text { Narrow rang } \\
\text { Multiplying } \\
\text { by a factor } \\
\alpha=30\end{array}$ & $\begin{array}{l}\text { quantization } \\
\text { Without } \\
\text { multiplying } \\
\text { by any factor }\end{array}$ \\
\hline $\begin{array}{l}\text { Technique of } \\
\text { Alex et al. }{ }^{11-13}\end{array}$ & $\begin{array}{l}\text { Signal1: "1.wav" } \\
\text { Signal2: "2.wav" } \\
\text { Signal3: "3.wav" }\end{array}$ & $\begin{array}{l}242688 \\
222464 \\
252800\end{array}$ & $\begin{array}{l}365568 \\
335104 \\
380800\end{array}$ \\
\hline $\begin{array}{l}\text { The proposed } \\
\text { Technique }\end{array}$ & $\begin{array}{l}\text { Signal1: "1.wav" } \\
\text { Signal2: "2.wav" } \\
\text { Signal3: "3.wav" }\end{array}$ & $\begin{array}{l}152460 \\
142296 \\
159236\end{array}$ & $\begin{array}{l}222464 \\
249816 \\
279556\end{array}$ \\
\hline
\end{tabular}

Table 3. Bits after compression in case of Narrow Range Quantization with or without multiplying the psychoacoustic model threshold by the factor $\alpha=30$.

\section{RESULTS AND DISCUSSION}

The results listed in Table 2, are in terms of bits before and after compression. They are obtained from the application of the proposed technique and that of Alex et al. ${ }^{11-13}$ to three different speech signals named "1.wav", "2.wav" and "3.wav", and sampled at $8 \mathrm{kHz}$.

Table 2 shows that the proposed speech compression technique outperforms the technique of Alex et al. ${ }^{11-13}$ In fact, the proposed technique yields higher compression ratios than the technique of Alex et al. ${ }^{11-13}$

According to figures 4 and 5, in the case of Narrow Range Quantization, the proposed technique and that of Alex et al. ${ }^{11-13}$ allows a degraded output speech signal to be obtained. To solve this problem, we multiplied the Psychoacoustic Model threshold by a factor $\alpha=30$.

Tables 3 and 4 list the results obtained from the application of the proposed technique and that of Alex et al. ${ }^{11-13}$ to the same speech signals ("1.wav", "2.wav", "3.wav"). Those results are obtained in case of narrow range quantization (with/without multiplying the psychoacoustic model threshold by the factor). They are in terms of bits before/after compression and PESQ.

According to Table 4, in case of Narrow range quantization, the PESQ values are worse when the psychoacoustic model threshold is not multiplied by any factor. This is justified by the fact that the proposed technique and that of Alex et al. ${ }^{11-13}$ both introduce severe degradations on the original speech signal after compression and de-compression (Figure $4 \mathrm{~b}$ and Figure 5 b). Table 4 shows that, when we multiply this threshold by a factor $\alpha=30$, the PESQ values are considerably improved for the two techniques. Therefore, the perceptual qualities of the reconstructed speech signals are ameliorated, and we have obtained acceptable PESQ values for the proposed technique. These PESQ values have been obtained by referring to the variation range of PESQ which is $[-0.5,4.5]$ as previously mentioned. Also, according to Table 4 and when the psychoacoustic model threshold is multiplied by a factor $\alpha=30$, the proposed technique gives lower PESQ values com- 


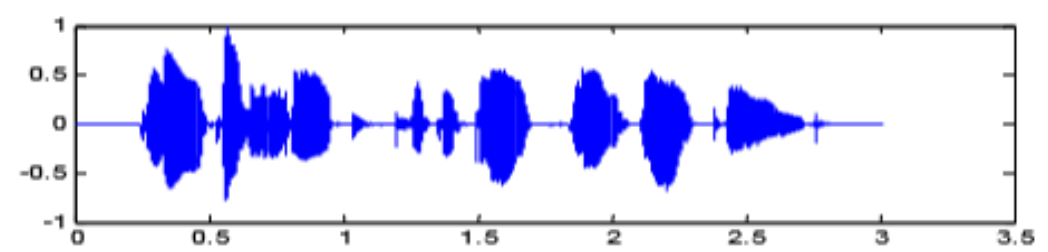

(a)

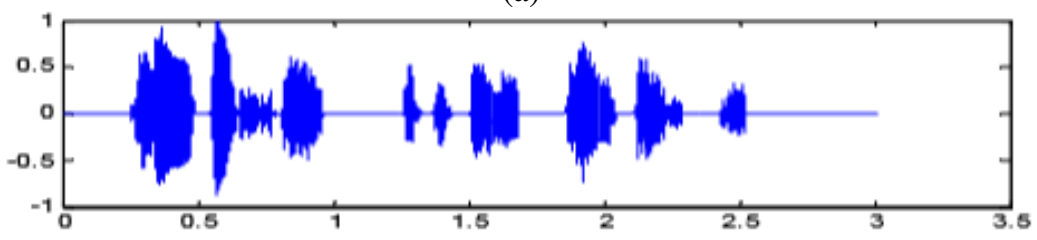

(b)

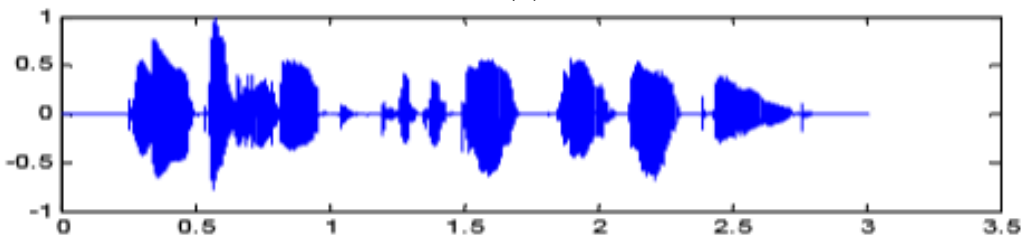

(c)

Figure 4. (a) Original Speech signal, (b) degraded output speech signal obtained from the compression system of Alex et al. ${ }^{11-13}$ (without multiplying the Psychoacoustic Model threshold by any factor), (c) output speech signal from the compression system of Alex et al. ${ }^{11-13}$ (with multiplying the Psychoacoustic Model threshold by $\alpha=30$ ).

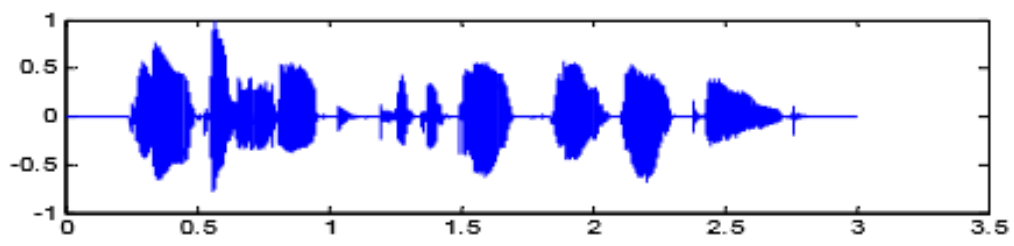

(a)

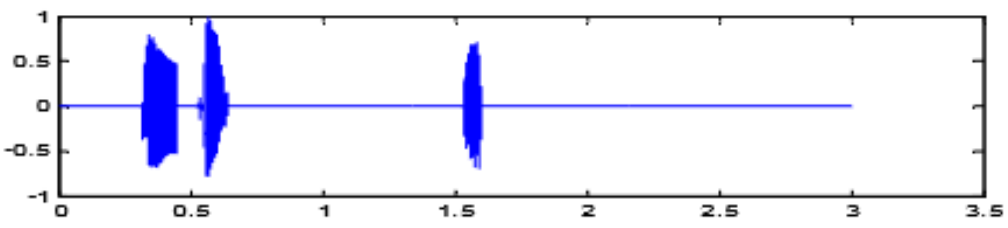

(b)

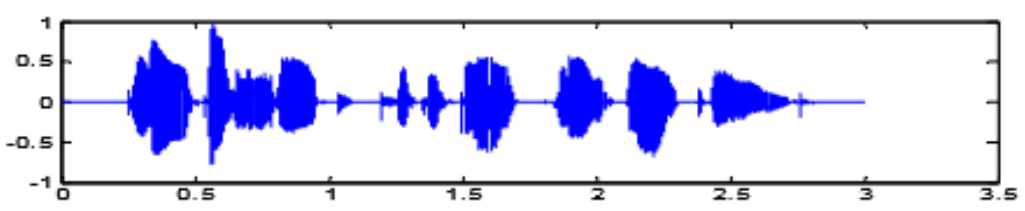

(c)

Figure 5. (a) Original Speech signal, (b) degraded output speech signal obtained from the proposed compression system (without multiplying the Psychoacoustic Model threshold by any factor), (c) output speech signal from the proposed compression system with multiplying the Psychoacoustic Model threshold by $\alpha=30$.

pared to the technique of Alex et al. ${ }^{11-13}$ This is justified by the fact that our proposed technique used a filter bank with just two filters while the MDCT filter bank employed by Alex et al. ${ }^{11-13}$ used 32 filters. Consequently, when using the MDCT filter bank, we had more precision and the error between the original and the reconstructed signals was reduced. Moreover, compared to the filter-bank designed using optimization, ${ }^{14,15}$ the MDCT Filter Bank with 32 filters allows for more flexibility and adaptability when analysing the frequency components of the speech signal to be compressed. Therefore, when the speech signal is compressed using the proposed technique, some significant frequency components are suppressed. However, they are conserved when using the compression technique of Alex et al. ${ }^{11-13}$ This is why the PESQ values obtained by the proposed compression technique are lower than those obtained by the compression technique of Alex et al. ${ }^{11-13}$ However, our proposed technique yields better compression ratios than the technique of Alex et al. ${ }^{11-13}$ Furthermore, it presents acceptable perceptual qualities for the reconstructed speech signals. Hence, the proposed technique ensures a compromise between 


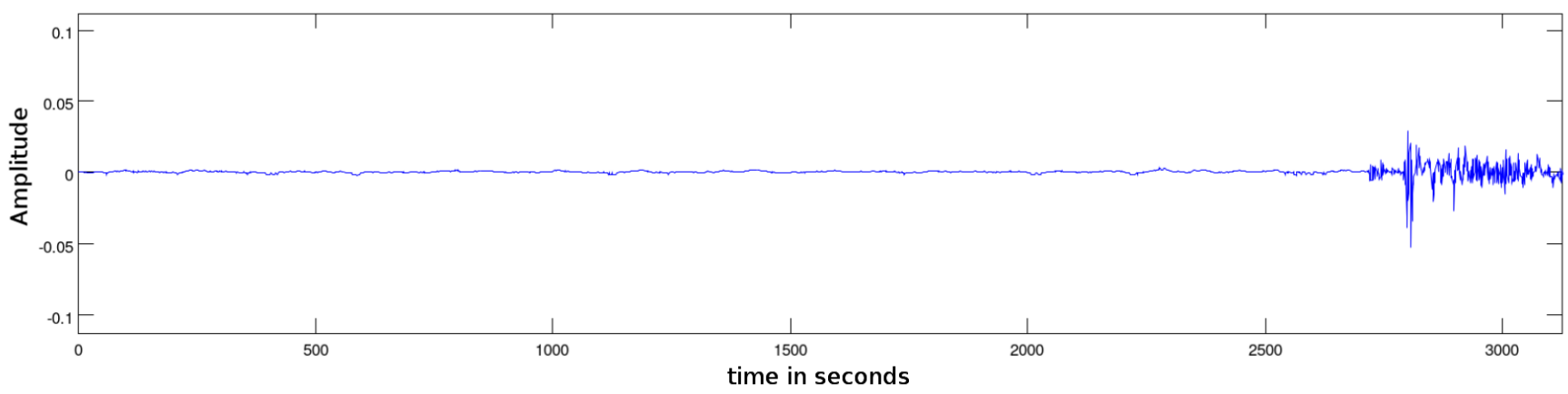

(a)

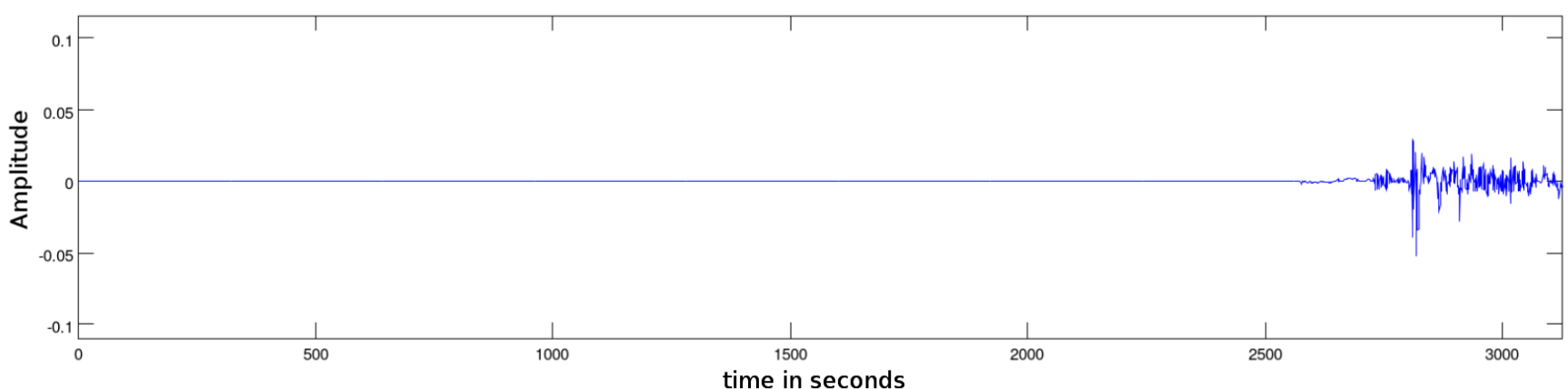

(b)

Figure 6. (a) The beginning (zoomed) of the original speech signal, (b) the beginning (zoomed) of the corresponding reconstructed speech signal.

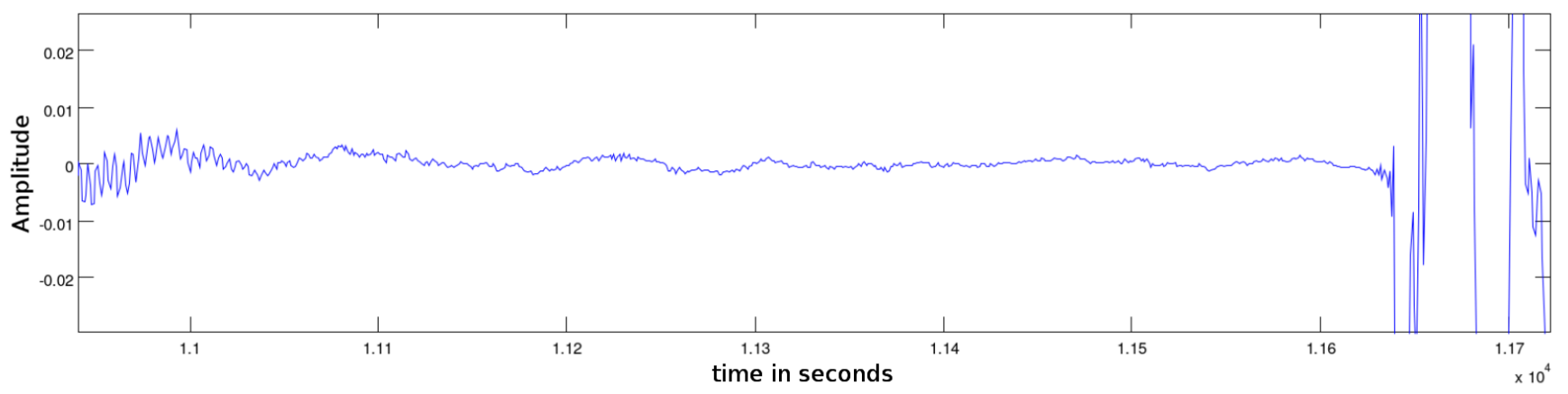

(c)

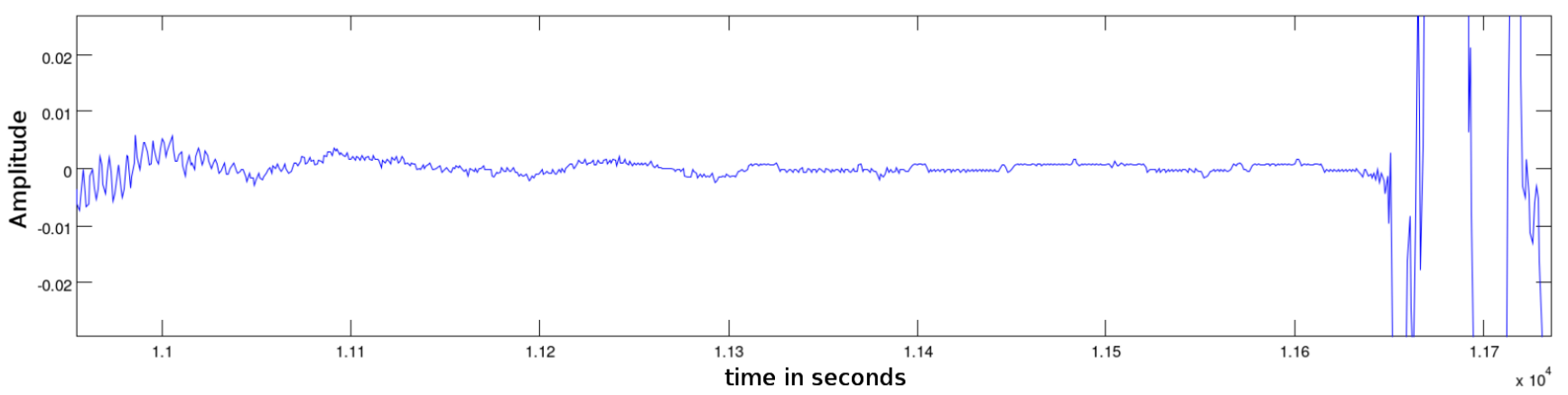

(d)

Figure 7. (c) The middle (zoomed) of the original speech signal, (d) the middle (zoomed) of the corresponding reconstructed speech signal.

a good compression ratio and acceptable perceptual qualities of the reconstructed speech signals.

Figures 6, 7 and 8 show three different zoomed parts of the original speech signal and the reconstructed one obtained after both compression and de-compression by using the proposed speech compression technique.
According to these Figures, the silent zones are practically zeroed while the others have reached their threshold. This is due to the application of quantization. Also, in the case of narrow range quantization, the proposed speech compression technique and that of Alex et al. ${ }^{11-13}$ provides reconstructed speech signals that are delayed with a little constant delay 


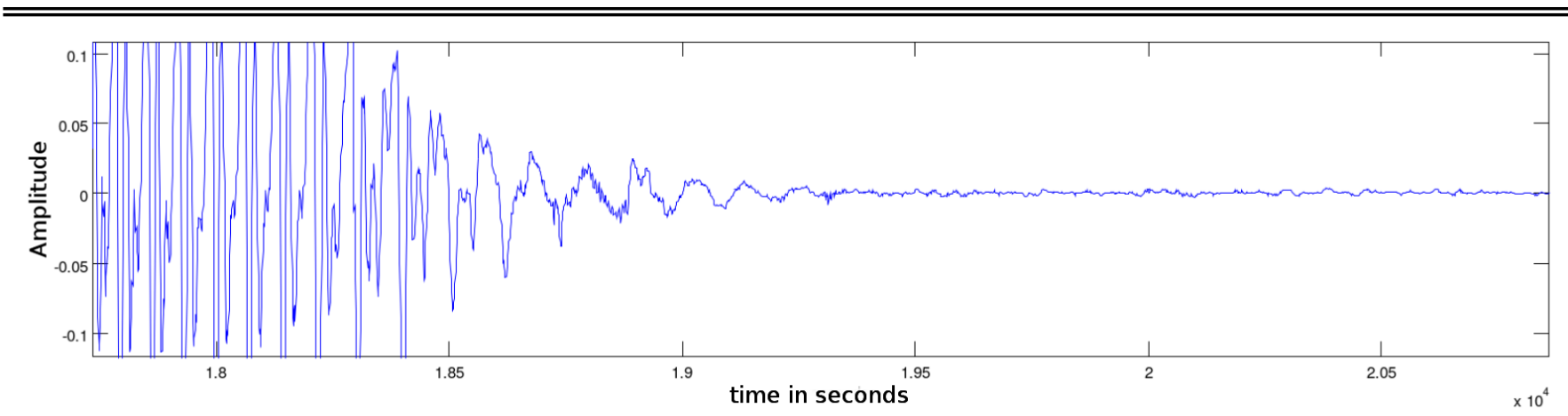

(e)

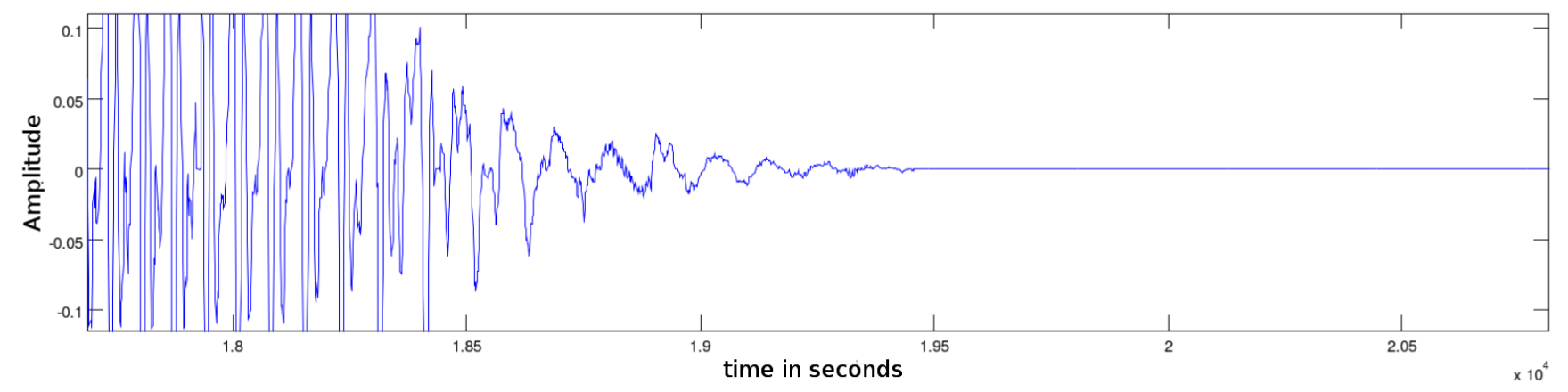

(f)

Figure 8. (e) The end (zoomed) of the original speech signal, (f) The end (zoomed) of the corresponding reconstructed speech signal.

Table 4. PESQ values of the reconstructed speech signal in case of Narrow range Quantization with or without multiplying the psychoacoustic model threshold by the factor $\alpha=30$.

\begin{tabular}{|c|c|c|c|}
\hline $\begin{array}{l}\text { The } \\
\text { Compression } \\
\text { Technique }\end{array}$ & Signal & $\begin{array}{l}\text { Narrow ra } \\
\text { quantizat } \\
\text { Multiply } \\
\text {-ing by a } \\
\text { factor } \\
\alpha=30\end{array}$ & $\begin{array}{l}\text { ge } \\
\text { n } \\
\text { Without } \\
\text { multiply- } \\
\text { ing by any } \\
\text { factor }\end{array}$ \\
\hline $\begin{array}{l}\text { Technique of } \\
\text { Alex et al. }{ }^{11-13}\end{array}$ & $\begin{array}{l}\text { Signal1: "original1.wav" } \\
\text { Signal2: "nhwy_jrmlr.wav" } \\
\text { Signal3: "1.wav" } \\
\text { Signal4: "3.wav" } \\
\text { Signal5: "5.wav" }\end{array}$ & $\begin{array}{l}4.28 \\
4.20 \\
3.52 \\
3.85 \\
3.70\end{array}$ & $\begin{array}{l}2.07 \\
2.22 \\
1.58 \\
2.07 \\
1.53\end{array}$ \\
\hline $\begin{array}{l}\text { The proposed } \\
\text { Technique }\end{array}$ & $\begin{array}{l}\text { Signal1: "original1.wav" } \\
\text { Signal2: "nhwy_jrmlr.wav" } \\
\text { Signal3: "1.wav" } \\
\text { Signal4: "3.wav" } \\
\text { Signal5: "5.wav" }\end{array}$ & $\begin{array}{l}3.28 \\
3.43 \\
2.43 \\
2.81 \\
2.48\end{array}$ & $\begin{array}{l}1.59 \\
1.66 \\
0.55 \\
0.84 \\
0.59\end{array}$ \\
\hline
\end{tabular}

when compared to the original speech signals. Consequently, we have suppressed this delay only for our proposed technique. We obtained the following results in terms of Bits before and after Compression, SNR, PSNR and NRMSE (Table 5).

Those results (Table 5) show that the values of SNR, PSNR and NRMSE obtained by the proposed technique are considerably better than those obtained by the technique of Alex et al. ${ }^{11-13}$ This is due to the delay that exists between the original signal and the reconstructed one obtained by the compression system of Alex et al. ${ }^{11-13}$ The values of SNR, PSNR and NRMSE that were obtained by the compression technique are good. Therefore, the output speech signals from the proposed speech compression system are in line with acceptable perceptual qualities. Moreover, in term of Compression Ratios, the proposed technique permits to obtain better Compression Ratios compared to the technique of Alex et al. ${ }^{11-13}$

\section{CONCLUSION}

In this paper, a new speech compression technique is proposed. It applies a Psychoacoustic Model and a general approach for Filter Bank Design using optimization. The role of the psychoacoustic modelling is to determine which parts of the speech signal to remove without suffering the loss of sound quality to the human ear. It is evaluated and compared with a compression technique based on a psychoacoustic model and MDCT filter bank that has 32 filters. This evaluation is performed by calculating bits before and after compression, SNR, PSNR, NRMSE and PESQ. The obtained results from this evaluation show that the proposed technique outperforms the second compression technique (based on a Psychoacoustic Model and MDCT filter Bank) in terms of Bits after compression and compression ratio. In fact, the proposed technique permits to have higher values of the compression ratio compared to the second compression technique. Furthermore, the proposed compression yields reconstructed speech signals with acceptable perceptual qualities. This is justified by the obtained values of SNR, PSNR and NRMSE and PESQ.

\section{ACKNOWLEDGEMENT}

We would like to thank all the people who contributed in some way to this work which was supported by CRTEn (Center of Researches and Technologies of Energy of Borj Cedria, Tunis) and the Ministry of Higher Education and Scientific Research of Tunisia.

\section{REFERENCES}

1 Xie, N., Dong, G., and Zhang, T. Using lossless data compression in data storage systems: not for saving space, 
Table 5. SNR, PSNR, NRMSE, Bits before/after Compression for the proposed compression technique and that of Alex et al.11-13 in case of Narrow range quantization.

\begin{tabular}{||l|l|c|c|c|c|c||}
\hline The Compression Technique & Signal & SNR & PSNR & NRMSE & Bits before Compression & Bits after Compression \\
\hline Technique of Alex et al. "13 & Signal1: "original1.wav" & -0.89 & 17.25 & 1.10 & 299008 & 192128 \\
& Signal2: "nhwy_jrmlr.wav" & -1.68 & 15.61 & 1.21 & 672000 & 439872 \\
& Signal3: "1.wav" & -3.68 & 11.90 & 1.52 & 373152 & 242688 \\
& Signal4: "3.wav" & -3.76 & 14.92 & 1.54 & 345680 & 222464 \\
& Signal5: "5.wav" & -4.36 & 12.54 & 1.65 & 385088 & 252800 \\
\hline The proposed Technique & Signal1: "original1.wav" & 16.83 & 34.93 & 0.14 & 299008 & 149616 \\
& Signal2: "nhwy_jrmlr.wav" & 15.73 & 33.00 & 0.16 & 672000 & 340792 \\
& Signal3: "1.wav" & 15.73 & 31.29 & 0.16 & 373152 & 187020 \\
& Signal4: "3.wav" & 17.43 & 36.10 & 0.13 & 345680 & 174552 \\
& Signal5: "5.wav" & 14.17 & 31.08 & 0.19 & 385088 & 195332 \\
\hline
\end{tabular}

IEEE Transactions on Computers, 60 (3), 335-345, 2011. https://dx.doi.org/10.1109/TC.2010.150

2 Gibson, J. D. Speech coding methods, standards, and applications, IEEE Circuits and Systems Magazine, 5 (4), 30-49, 2005. https://dx.doi.org/10.1109/MCAS.2005.1550167

3 Riadh A., Salim S., Said G., Ali C. and Taleb Ahmed A. Novel Detection Algorithm of Speech Activity and the impact of Speech Codecs on Remote Speaker Recognition System, WSEAS Transactions on Signal Processing. E-ISSN: 2224-3488, 2014.

4 Junejo, N., Ahmed, N., Unar, M. A., and Rajput, A. Q. K. Speech and image compression using discrete wavelet transform, IEEE symposium on advances in wired and wireless communication, 2005, pp. 45-48. https://dx.doi.org/10.1109/SARNOF.2005.1426508

5 Arif, M., and Anand, R. S. Turning point algorithm for speech signal compression, International Journal of Speech Technology, 2012. https://dx.doi.org/10.1007/s10772-0129151-7

6 Gersho, A. Speech coding. in A. N. Ince (Ed.), Digital speech processing, pp. 73-100. Boston: Kluwer Academic, 1992.

7 Gersho, A. Advance in speech and audio compression, Proceedings of the IEEE, 1994, 82 (6), 900-918. https://dx.doi.org/10.1109/5.286194

8 Agbinya, J. I. Discrte wavelet transform techniques in speech processing, IEEE Tencon digital signal processing applications proceedings, New York: IEEE, 1996, pp. 514-519. https://dx.doi.org/10.1109/TENCON.1996.608394

9 Gershikov, E., and Porat, M. On color transforms and bit allocation for optimal subband image compression. Signal Processing, Image Communication, 22, 1-18, 2007. https://dx.doi.org/10.1016/j.image.2006.09.003
10 Boussalmi, S., Aloui, N., Cherif, A.. Adaptive Speech Compression Based on Discrete Wave Atoms Transform, International Journal of Electrical and Computer Engineering (IJECE), 6 (5), October 2016, pp. 2150-2157.

11 W.A.V.S. Compression, http://www a amusings .

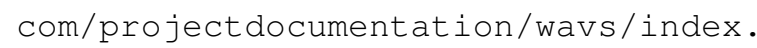
html.

12 http://www.360doc.com/content/05/0810/ 17/641_5382.shtml

13 Giron-Sierra, J. M., Digital Signal Processing with Matlab Examples, Springer, XXXVII, 622 2017. http:// www.springer.com/us/book/9789811025334 https://dx.doi.org/10.1007/978-981-10-2534-1

14 Moazzen, I., Agathoklis, P. A General Approach for Filter Bank Design Using Optimization, Technical Report, 2014, http://www.ece.uvic.ca/ imanmoaz/publications.htm.

15 https: / / www. mathworks.com/ matlabcentral/fileexchange/ 40128-filter-bank-design

16 Talbi, M., Barnoussa, C., Cherif, A. Speech Compression based on Psychoacoustic Model and A General Approach for Filter Bank Design using Optimization; ACIT'2013, 2013.

17 Talbi, M., Speech enhancement based on stationary bionic wavelet transform and maximum a posterior estimator of magnitude-squared spectrum, International Journal of Speech Technology, 20 (1), pp. 75-88, March 2017. https://dx.doi.org/10.1007/s10772-016-9388-7

18 Shrawanka, U., Voice Activity Detector and Noise Trackers for Speech Recognition System in Noisy Environment, International Journal of Advancements in Computing Technology, 2 (4), 2010. https://dx.doi.org/10.4156/ijact.vol2.issue4.11 